\title{
Complex Regional Pain Syndrome and Dysautonomia in a 14-Year-Old Girl Responsive to Therapeutic Plasma Exchange
}

\author{
Jeanne E. Hendrickson, ${ }^{1,2 \star}$ Emma T. Hendrickson, ${ }^{3}$ Eric A. Gehrie, ${ }^{1}$ Davinder Sidhu, ${ }^{1}$ Gerd Wallukat, ${ }^{4,5}$ \\ Ingolf Schimke, ${ }^{4}$ and Christopher A. Tormey ${ }^{1,6}$ \\ ${ }^{1}$ Department of Laboratory Medicine, Yale University School of Medicine, New Haven, Connecticut \\ ${ }^{2}$ Department of Pediatrics, Yale University School of Medicine, New Haven, Connecticut \\ ${ }^{3}$ Adams School, Guilford, Connecticut \\ ${ }^{4}$ Berlin Cures $\mathrm{GmbH}$, Berlin, Germany \\ ${ }^{5}$ Max Delbrück Center for Moleculare Medicine, Berlin, Germany \\ ${ }^{6}$ VA Connecticut Healthcare System, West Haven, Connecticut
}

\begin{abstract}
Reflex sympathetic dystrophy, also known as complex regional pain syndrome (CRPS), has recently been shown to be associated with autoantibodies against $\beta 2$-adrenergic and muscarinic M2 receptors. In addition to pain and sudomotor/vasomotor symptoms, dysautonomia is also observed in a subset of CRPS patients. Despite its severity, there are few effective therapies for CRPS described to date. We report a case of a 14-year-old girl with CRPS of her right leg and dysautonomia (gastroparesis, postural tachycardia) refractory to multiple therapies, successfully treated with therapeutic plasma exchange (TPE) with albumin replacement. The patient, who has serum anti $\beta 2$ adrenergic and muscarinic M2 receptor autoantibodies in addition to nicotinic acetylcholine receptor ganglionic autoantibodies, underwent an initial course of five TPEs over a 2-week period. She demonstrated a clinical response to TPE as manifested by a rapid improvement in her fatigue and gastroparesis, with a gradual yet significant improvement in her leg pain and sudomotor/vasomotor flares. Following the loading procedures, the patient was treated with rituximab. She continues to require periodic TPE to maintain a remission, with additional immunosuppression being considered long term. Although further studies are needed, TPE (in combination with immunosuppression) may be an appropriate therapy for CRPS patients with detectable autoantibodies, as it is for better characterized diseases with autoantibodies against neuronal surface receptors such as myasthenia gravis or Lambert Eaton myasthenic syndrome. J. Clin. Apheresis 00:000-000, 2015. (c) 2015 Wiley Periodicals, Inc.
\end{abstract}

Key words: CRPS; RSD; therapeutic plasma exchange; immunosuppression

\section{INTRODUCTION}

Complex regional pain syndrome (CRPS), previously referred to as Reflex Sympathetic Dystrophy (RSD), is a debilitating disease. Despite being described $>100$ years ago, the etiology of this disease has historically been poorly understood [1]. Symptoms include severe pain, allodynia, vasomotor, and trophic changes of the affected area. Upper extremities tend to be affected in adult cases of CRPS, whereas lower extremities are affected more often in childhood cases. A traumatic event, such as a soft tissue injury or a fracture, precedes many cases. Given the varying presentations and courses of CRPS, this disease has been categorized into "type I" or "type II," with type I having no nerve lesion identified and type II being termed "causalgia" due to a specific nerve injury.

It is likely that there are further subcategories for CRPS, though they are not well defined. Many patients with CRPS have systemic symptoms, as reviewed by Schwartzman [2]. Such symptoms may affect multiple organ systems, including respiratory, cardiovascular, gastrointestinal, genitourinary, and others. Dysautonomic symptoms may be a prominent systemic feature in some CRPS patients. Like extremity CRPS symptoms, however, the etiology of these systemic symptoms has also historically been poorly understood.

CRPS is increasingly being appreciated to have an autoimmune etiology. Early studies suggesting a female preponderance and an HLA association [3,4] led to a search for autoantibodies. In 2004, Blaes et al. described autoantibodies against the autonomic nervous system in a subset of CRPS patients [5]. In 2009, these

*Correspondence to: Jeanne E. Hendrickson, Yale University Department of Laboratory Medicine and Pediatrics, 330 Cedar Street, Clinic Building 405, P.O. Box 208035, New Haven, CT 06520-0835, USA. E-mail: jeanne.hendrickson@yale.edu.

Received 13 March 2015; Accepted 1 May 2015

Published online 00 Month 2015 in Wiley Online Library (wileyonlinelibrary.com).

DOI: $10.1002 /$ jca. 21407 
autoantibodies were further determined to bind to surface receptors of primary cultured autonomic sympathetic and myenteric plexus neurons, including differentiated neurons with a cholinergic phenotype [6]. A landmark study published in 2011 by Kohr et al. identified the autoantibody target as $\beta 2$-adrenergic and muscarinic M2 receptors, with binding against the second extracellular loop described [7]. These antibodies were further shown to be functionally active via a cardiomyocyte bioassay [7]; another laboratory also reported functionally active autoantibodies against alpha-1a receptors in patients with longstanding CRPS [8]. Passive transfer experiments of sera from humans with CRPS into mice led to clinical and laboratory features resembling the human disease $[9,10]$, further supporting the pathologic nature of these detected autoantibodies.

Historically, few treatments for CRPS have been effective. An extensive review of potential treatments is summarized by Harden et al. [1]. However, few randomized controlled trials have been conducted on CRPS patients. In brief, a 2004 randomized trial of gabapentin showed a mild effect on pain in some CRPS patients [11]. In 2013, a randomized trial of the amino-bisphosphonate neridronate showed improvement in pain in some participants [12,13]. Ketamine infusions, nerve blocks, spinal cord stimulators, systemic analgesia, and other treatments have been shown to be partially effective in a subset of CRPS patients, but these therapies represent case series or anecdotal reports.

Immunomodulatory therapies for CRPS have only recently begun to be investigated. Steroid treatment has been show to help CRPS symptoms in case reports and small studies [14-16]. Anti-tumor necrosis factor (TNF) treatment has been reported to result in subtle improvements in pain in some CRPS patients $[17,18]$. Intravenous immunoglobulin (IVIG) has also recently been investigated: a case series of patients with chronic pain (including some with CRPS) treated with a trial of IVIG was published in 2002, with $50 \%$ of patients being shown to have a $30 \%$ reduction in their pain [19]. A case report published in 2005 showed transient improvement in CRPS symptoms in a single patient following IVIG infusion [20], and another case report published in 2013 showed significant improvement in a patient following high dose $(2 \mathrm{~g} / \mathrm{kg}$ ) IVIG infusion [21]. A small randomized controlled crossover trial showed partial improvement in pain and autonomic limb symptoms in patients with refractory CRPS following low dose $(0.5 \mathrm{~g} / \mathrm{kg})$ IVIG [22]; a larger randomized controlled trial of low dose IVIG in CRPS patients is currently ongoing [23].

Therapeutic plasma exchange (TPE), which serves as an effective adjunctive therapy for other neuroimmune disorders that are mediated in part by autoanti- bodies against surface receptors [24], can transiently reduce circulating $\beta 2$-adrenergic and muscarinic M2 receptor autoantibodies. To the degree that these antibodies are related to CRPS symptoms [25], antibody removal would be expected to lead to at least partial symptomatic improvement. Two case series were published in 2014 to 2015 on a total of 8 CRPS patients treated with TPE; 7 of these patients had at least a partial response in terms of pain relief, improvement of autonomic dysfunction, or improvement in cognition $[25,26]$. Here, we report the use of TPE in a 14-yearold girl with longstanding CRPS and dysautonomia (associated with the detection of several autoantibodies), resulting in significant symptomatic improvement.

\section{CASE REPORT}

A 14-year-old girl, with a 10-year history of worsening right knee pain and gastrointestinal dysmotility, presented to an urgent care clinic with severe (9 out of 10 on the numerical rating scale) [27] right knee pain after a minor fall in school gym class. Imaging, including a plain X-ray (XR) and an magnetic resonance imaging (MRI) scan, was normal. The patient was placed in a knee immobilizer and put on crutches, due to an inability to put any weight on her leg without severe pain. Intermittent color changes of her entire right leg from the upper thigh to the mid portion of the dorsum of her right foot were observed (Figs. 1A-1C), as were intermittent temperature changes ranging from "ice cold" to episodes of isolated sweating. The location of her most severe pain, the medial aspect of her right knee, was noted to be bruised approximately 2 weeks after the injury (Fig. 1D). The patient was referred to a tertiary care center, where her exam showed allodynia in the distribution of her right leg where she was also experiencing pain and vasomotor symptoms. Her reflexes were normal, evaluation of right leg strength was limited by severe pain, and fine bilateral hand tremors were noted. Her pupils were dilated but responsive to light. Based on her history and clinical presentation, she was diagnosed with CRPS.

The patient was serially treated with the following medications: nonsteroidal anti-inflammatory drugs (ibuprofen, naproxen), acetaminophen, codeine, gabapentin, amitryptaline, tramadol, prednisone, and pregabalin. Despite these therapies, she continued to rate her pain as a 9 out of 10 and was unable to put any weight on her right leg. She was also treated with twice weekly physical therapy, including desensitization therapy, without any improvement in her pain or vasomotor limb symptoms. Likewise, cognitive behavior therapy did not impact her pain. She had no improvement in her symptoms with local therapy, including 
A.

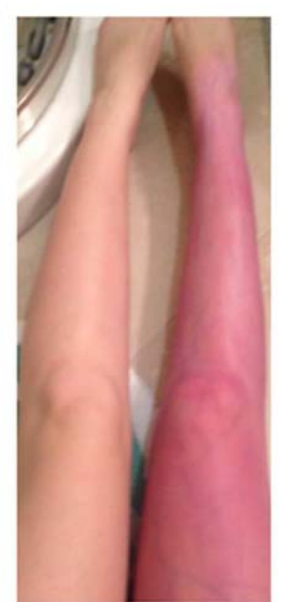

B.

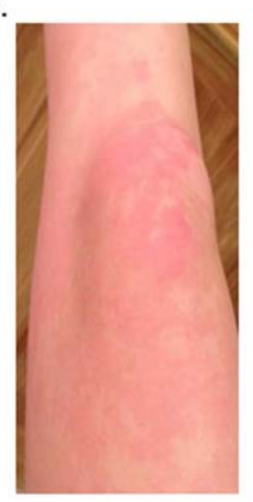

C.

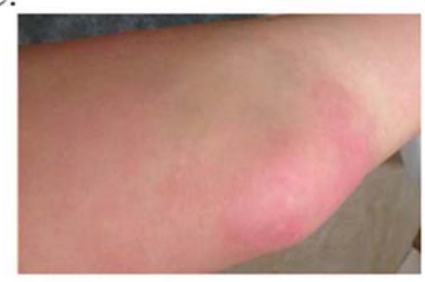

D.

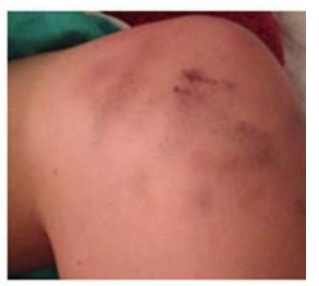

Fig. 1. Lower extremity images. A-C, Depict discoloration of the patient's right lower extremity during sudomotor/vasomotor flares. D, Depicts bruising of the medial aspect of the patient's right knee (the location of her most severe pain).

transcutaneous electrical nerve stimulation (TENS), diclofenac gel, and capsaicin cream.

In addition to her right leg pain, she began to experience worsening dysautonomic symptoms 1 to 2 months after her injury, including gastroparesis, constipation, tachycardia, and orthostatic hypotension. She also developed significant fatigue and dizziness, persistent discoloration of her leg during flares, and lack of hair and toe nail growth in the affected limb. She was ultimately hospitalized for one week due to an inability to tolerate any solid foods, with an upper endoscopic examination revealing redundant antral tissue (Fig. 2) and delayed opening of the pylorus to the endoscope. An upper gastrointestinal study showed delayed emptying of barium through the pylorus, with no physical obstruction noted. Erythromycin therapy slightly improved her GI symptoms, although she continued to experience stomach pain with solid food intake as well as constipation. Neither metoclopramide nor pyridostigmine bromide improved her GI motility. An electrocardiogram completed during her hospitalization, ordered to evaluate new chest pain, showed sinus tachycardia with a baseline heart rate of 110; an echocardiogram showed a structurally normal heart with continuous tachycardia. At that time, her blood pressure when supine was 110/70 with a heart rate of 110 beats/min; within 1 min of standing her blood pressure was 90/60 with a heart rate of 130 to 140 beats/min. A brain MRI with and without contrast was normal. A dual-energy X-ray absorptiometry (DEXA) scan was normal for age.

Given worsening dysautonomic symptoms, the patient's serum was sent to Mayo Medical Laboratories (Rochester, MN) for an Autoimmune Dysautonomia evaluation. The testing revealed an acetylcholine receptor ganglionic neuronal antibody $(0.08 \mathrm{nmol} / \mathrm{L}$; reference value $\leq 0.02$ ).

Serum was also sent to Berlin Cures GmbH (Berlin, Germany), and the immunoglobulin fraction was tested at a dilution of 1:40. In the bioassay performed, functional autoantibodies against G-protein coupled receptors change the rate of spontaneously beating neonatal rat cardiomyocytes like the corresponding agonists; the effects of autoantibodies can be blocked by the corresponding receptor antagonists. Two different
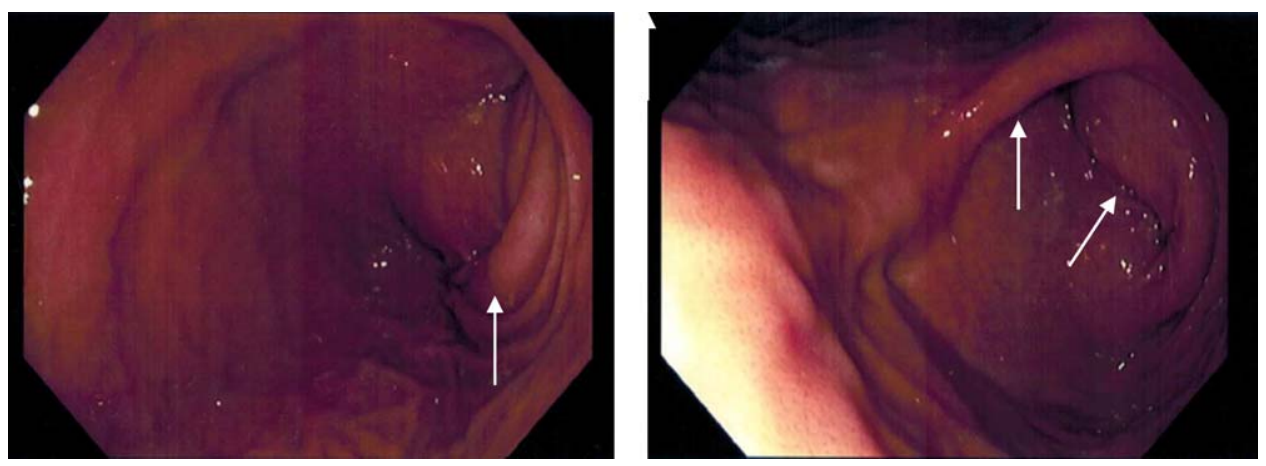

Fig. 2. Upper endoscopy, with evidence of a thickened antrum/pylorus. 

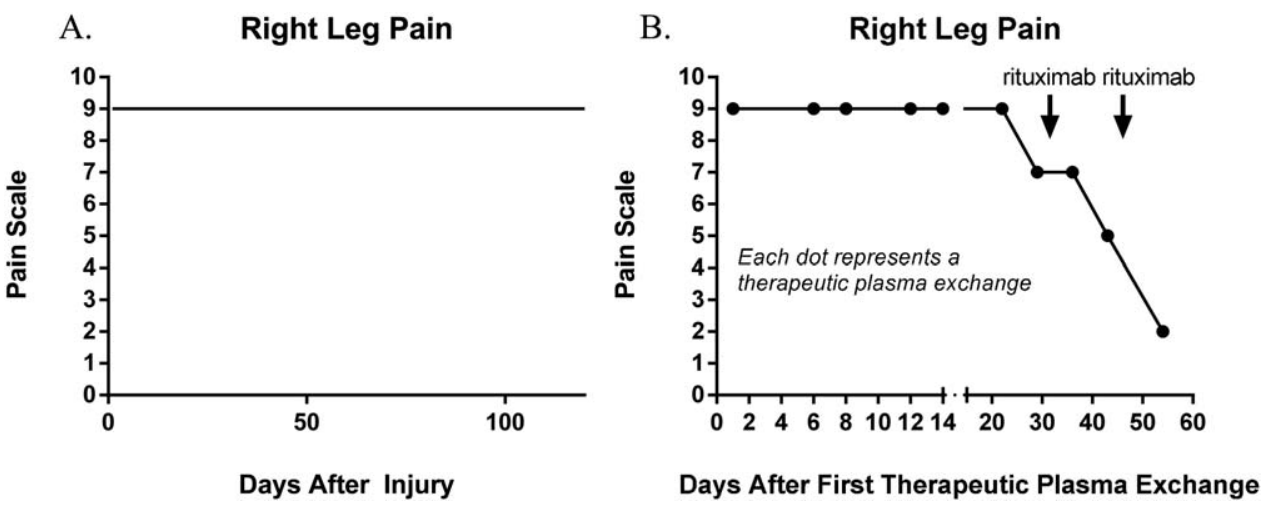

Fig. 3. Scale of right leg pain, before (A) and after (B) the initiation of TPE.

autoantibodies were identified in this patient's serum. One was directed against the $\beta 2$-adrenergic receptor, and increased the beating rate of the cardiomyocytes $(\Delta$ beats $/ \min =26.0)$. This effect was blocked by the specific antagonist ICI118.551 $(0.1 \mu \mathrm{M}),(\Delta$ beats/min $=0.0$ ). The other autoantibody detected was directed against the muscarinic M2 receptor and caused a negative chronotropic effect ( $\Delta$ beats $/ \mathrm{min}=-20.7)$. This effect was blocked by $1 \mu \mathrm{M}$ atropine ( $\Delta$ beats/min $=0.0)$. The calculated cut off value of normal was $\Delta$ beats $/ \min =7.2$. Moreover, the functional activity of the detected autoantibodies was neutralized by short overlapping peptides corresponding to the second extracellular loop of both receptors; the autoantibodies recognized in both receptors an epitope localized in the $\mathrm{N}$-terminal portion. Detailed information concerning IgG preparation, preparation and culture of neonatal rat cardiomyocytes, bioassay handling, and antibody differentiation has previously been described [28].

Other laboratory values, including thyroid function tests, a complete metabolic panel, a complete blood count, a PT/PTT, a vWF panel, an antinuclear antibody (ANA), a rheumatoid factor, a C-reactive protein (CRP), and a cyclic citrulinated peptide, were all within normal limits. Anti-phosphatidylserine $\operatorname{IgA}$ and $\operatorname{IgG}[29,30]$ were both negative.

TPE was initiated in light of the patient's refractory leg symptoms, worsening dysautonomia, the detection of autoantibodies, and two recently published case series $[25,26]$. All other previous therapies had been discontinued at the time of initiation of TPE, including physical therapy as well as cognitive behavioral therapy. An initial intensive schedule of five TPE procedures over a 14day period were completed using a Cobe Spectra Apheresis System (TerumoBCT, Lakewood, CO), with each procedure exchanging one plasma volume. Albumin with calcium gluconate was used as the replacement fluid and the total fluid balance was set to $110 \%$ to address the patient's low baseline blood pressure. After the second TPE, the patient's fatigue and nausea began to improve. Additionally, the patient's right leg stopped flaring (i.e., sweating or turning bright red or ice cold) after the second procedure. After the third procedure, she could eat solid foods without difficulty and she began diuresing and having regular bowel movements. After the fifth procedure, her dizziness began to improve. After completion of the induction TPE the patient's right leg hair and toenails, which had not grown at all in 4 months, began growing again. Furthermore the bruising of her right knee, which had been present for 4 months, resolved. The patient tolerated all loading TPEs without significant incident, although some perioral tingling was noted at the completion of procedures one and two.

Maintenance procedures were initially planned to be monthly, though 8 days after the patient's last TPE her nausea and GI dysmotility returned as did her dizziness and fatigue. Her leg pain, which had just begun to subside slightly, also worsened again. The symptoms were significantly improved once the intervals between TPE treatments were shortened. This pattern of partial symptom recurrence within 7 to 8 days of the past procedure and significant improvement within 12 to $24 \mathrm{~h}$ after the next procedure persisted for 2 months. Over the course of time, the patient's right leg pain slowly but very significantly improved (Fig. 3, right). Of note, she had rated her pain as a 9 out of 10 for a full 120 days before the initiation of TPE (Fig. 3, left).

Rituximab, at a dose of $500 \mathrm{mg} / \mathrm{m}^{2}$, was administered twice ( 2 weeks apart), and the intervals between TPE procedures were again attempted to be spaced further. At the time of this report (5 weeks after the first rituximab dose), TPE was still being required weekly to control CRPS and GI dysmotility symptoms. The patient's cardiovascular dysautonomic symptoms improved somewhat after rituximab, with less hypotension but persistent tachycardia. Autoantibody testing has not been repeated as of this writing.

\section{DISCUSSION}

TPE was effective in symptomatic relief for this 14year-old girl with CRPS, dysautonomia, and 
autoantibodies against $\beta 2$-adrenergic and muscarinic M2 receptors. Her CRPS was longstanding, likely having been present for over a decade. However, it was not diagnosed until a minor injury led to severe pain and classic sudomotor/vasomotor findings. This patient's disease, which involved not only her right leg (with pain rated as a 9 out of 10 for 4 months) but also her cardiovascular system (postural tachycardia/ fatigue), and her GI system (gastroparesis/dysmotility), was refractory to all other therapies attempted before plasmapheresis. TPE significantly improved each of her symptoms, just as it has recently been described to improve the symptoms of other patients with CRPS [25,26].

It is not surprising that TPE is effective for CRPS with $\beta 2$-adrenergic and/or muscarinic M2 receptor autoantibodies, given that TPE is currently a first or second line therapy for other neuroimmune diseases involving surface binding autoantibodies such as myasthenia gravis or Lambert-Eaton myasthenic syndrome [24]. Removal of the patient's autoantibodies presumably led to her symptomatic improvement, though repeat autoantibody testing was not completed given logistic difficulties. It cannot be ruled out, however, that removal of other substances such as inflammatory cytokines [31-33] may also have contributed to her improvement.

At the present time it is not known what percentage of CRPS patients have detectable autoantibodies, with few patients [7] having been tested for $\beta 2$-adrenergic or muscarinic M2 receptor autoantibodies. To the authors' knowledge, and after extensive investigations among CLIA-certified reference centers, there appear to be no laboratories in the United States that offer these tests in a clinical setting. The assays [34] are technically challenging and labor intensive, with the bioassay platform utilized for this patient's serum involving beating rat cardiomyocytes. Of note, our patient also tested positive for nicotinic ganglionic receptor autoantibodies; this is a radioimmunoprecipitation assay using iodine 125-labeled epibatinine complexed to the $\alpha 3$ acetylcholine receptor solubilized from a human neuroblastoma cell line. It is plausible that these autoantibodies may also be contributing to our patient's GI dysmotility and dysautonomia.

A subset of CRPS patients exhibit dysautonomic symptoms, and there is an increasing appreciation for the potential contributor of autoimmunity to dysautonomia in postural tachycardia (POTS), GI dysmotility, and autonomic ganglionopathy $[2,35]$. Li et al. recently described $\beta 1$-adrenergic autoantibodies in $14 / 14$ patients with POTS, with $\beta 2$-adrenergic autoantibodies being detected in approximately half of these patients [36]; previous studies have also suggested a potential autoimmune component to this disease with some patients also having ganglionic acetylcholine receptor antibodies [37,38]. These same ganglionic receptor autoantibodies, primarily to the $\alpha 3$ subunit of nicotinic acetylcholine receptors, have also been described to contribute to GI dysmotility [37,39-41] and autoimmune autonomic ganglionopathy $[40,42,43]$. Of 15,000 patients tested by the Mayo Clinic Laboratories, ganglionic receptor autoantibodies were detected in $<1 \%$. High levels $(\geq 1 \mathrm{nmol} / \mathrm{L})$ were associated with pandysautonomia. Medium levels $(0.10-0.99 \mathrm{nmol} / \mathrm{L})$ were associated with peripheral neuropathies, dysautonomia, or encephalopathy. Low levels (0.03-0.09 nmol/L), such as those detected in this patient, have typically been associated with nonautoimmune disorders or peripheral neuropathies [44].

Our patient has required weekly TPE to control her symptoms, thus prompting a trial of rituximab. To the authors' knowledge, utilization of rituximab for CRPS has never previously been published in the medical literature, though it has been used successfully in dysautonomia $[42,45]$ and other autoimmune and autoantibodymediated disorders $[46,47]$, including those responsive to plasmapheresis such as myasthenia gravis $[48,49]$. Longer term follow-up is needed before the efficacy of rituximab in this case can be commented on. Additional immunosuppression, potentially in combination with adjunctive therapies, will likely be necessary for this patient should frequent plasmapheresis continue to be needed post-rituximab for symptomatic relief.

As with any case report, it should be emphasized that a limitation of our manuscript is that we investigated only a single patient. It remains to be determined whether TPE may be an ideal first or second-line therapy for patients with a diagnosis of CRPS, or perhaps may be most applicable to those with CRPS and dysautonomic features. Identification of circulating autoantibody(ies) before TPE initiation would be ideal, though such an approach remains logistically challenging in the US. The role of long-term TPE for CRPS, both in this patient and in others, is not known at this point in time.

In sum, TPE with albumin replacement, with an initial intensive course of five procedures over 14 days followed by a periodic maintenance schedule, provided impressive symptomatic relief for our patient with severe, refractory CRPS and dysautonomia. This case, in combination with two recently published case series documenting symptomatic relief in adult CRPS patients treated with TPE $[25,26]$, suggest that this intervention - though not risk free-may be one of the more successful treatments described to date for this disease. Additional studies of the autoimmune nature of CRPS, including larger studies of TPE, are warranted.

\section{ACKNOWLEDGMENTS}

The authors thank the Apheresis Nurses at Yale-New Haven Hospital for their tremendous care of this patient. 
J.E.H. thanks many clinicians, colleagues, researchers, and friends for their invaluable input and/or care of this patient.

\section{AUTHOR CONTRIBUTIONS}

J.E.H., E.T.H., and C.A.T. wrote the manuscript, and all authors edited the manuscript and approved of the final version. G.W. and I.S. also contributed time and reagents for antibody testing.

\section{REFERENCES}

1. Harden RN, Oaklander AL, Burton AW, Perez RS, Richardson K, Swan M, Barthel J, Costa B, Graciosa JR, Bruehl S. Complex regional pain syndrome: practical diagnostic and treatment guidelines, 4th edition. Pain Med (Malden, Mass) 2013;14:180229.

2. Schwartzman RJ. Systemic complications of complex regional pain syndrome. Neurosci Med 2012;3:225-242.

3. van de Beek WJ, van Hilten JJ, Roep BO. HLA-dq1 associated with reflex sympathetic dystrophy. Neurology 2000;55:457-458.

4. van Rooijen DE, Roelen DL, Verduijn W, Haasnoot GW, Huygen FJ, Perez RS, Claas FH, Marinus J, van Hilten JJ, van den Maagdenberg AM. Genetic HLA associations in complex regional pain syndrome with and without dystonia. J Pain 2012; 13:784-789.

5. Blaes F, Schmitz K, Tschernatsch M, Kaps M, Krasenbrink I, Hempelmann G, Brau ME. Autoimmune etiology of complex regional pain syndrome (M. Sudeck). Neurology 2004;63:17341736.

6. Kohr D, Tschernatsch M, Schmitz K, Singh P, Kaps M, Schafer KH, Diener M, Mathies J, Matz O, Kummer W, Maihofner C, Fritz T, Birklein F, Blaes F. Autoantibodies in complex regional pain syndrome bind to a differentiation-dependent neuronal surface autoantigen. Pain 2009;143:246-251.

7. Kohr D, Singh P, Tschernatsch M, Kaps M, Pouokam E, Diener M, Kummer W, Birklein F, Vincent A, Goebel A, Wallukat G, Blaes F. Autoimmunity against the beta2 adrenergic receptor and muscarinic-2 receptor in complex regional pain syndrome. Pain 2011;152:2690-2700.

8. Dubuis E, Thompson V, Leite MI, Blaes F, Maihofner C, Greensmith D, Vincent A, Shenker N, Kuttikat A, Leuwer M, Goebel A. Longstanding complex regional pain syndrome is associated with activating autoantibodies against alpha-1a adrenoceptors. Pain 2014;155:2408-2417.

9. Goebel A, Leite MI, Yang L, Deacon R, Cendan CM, FoxLewis A, Vincent A. The passive transfer of immunoglobulin G serum antibodies from patients with longstanding complex regional pain Syndrome. Eur J Pain 2011;15:504.e501-506.

10. Tekus V, Hajna Z, Borbely E, Markovics A, Bagoly T, Szolcsanyi J, Thompson V, Kemeny A, Helyes Z, Goebel A. A CRPS-IgG-transfer-trauma model reproducing inflammatory and positive sensory signs associated with complex regional pain syndrome. Pain 2014;155:299-308.

11. van de Vusse AC, Stomp-van den Berg SG, Kessels AH, Weber WE. Randomised controlled trial of gabapentin in complex regional pain syndrome type 1 [isrctn84121379]. BMC Neurol 2004;4:13.

12. Varenna M, Adami S, Rossini M, Gatti D, Idolazzi L, Zucchi F, Malavolta N, Sinigaglia L. Treatment of complex regional pain syndrome type I with neridronate: a randomized, double-blind, placebo-controlled study. Rheumatology (Oxford) 2013;52:534542.
13. Varenna M, Adami S, Sinigaglia L. Bisphosphonates in complex regional pain syndrome type I: how do they work? Clin Exp Rheumatol 2014;32:451-454.

14. Braus DF, Krauss JK, Strobel J. The shoulder-hand syndrome after stroke: a prospective clinical trial. Ann Neurol 1994;36: 728-733.

15. Christensen K, Jensen EM, Noer I. The reflex dystrophy syndrome response to treatment with systemic corticosteroids. Acta Chir Scand 1982;148:653-655.

16. Atalay NS, Ercidogan O, Akkaya N, Sahin F. Prednisolone in complex regional pain syndrome. Pain Physician 2014;17:179185.

17. Eisenberg E, Sandler I, Treister R, Suzan E, Haddad M. Anti tumor necrosis factor - alpha adalimumab for complex regional pain syndrome type 1 (CRPS-I): a case series. Pain Pract 2013; 13:649-656.

18. Huygen FJ, Niehof S, Zijlstra FJ, van Hagen PM, van Daele PL. Successful treatment of CRPS 1 with anti-TNF. J Pain Symptom Manage 2004;27:101-103.

19. Goebel A, Netal S, Schedel R, Sprotte G. Human pooled immunoglobulin in the treatment of chronic pain syndromes. Pain Med (Malden, Mass) 2002;3:119-127.

20. Goebel A, Stock M, Deacon R, Sprotte G, Vincent A. Intravenous immunoglobulin response and evidence for pathogenic antibodies in a case of complex regional pain syndrome 1. Ann Neurol 2005;57:463-464.

21. Medlin F, Zekeridou A, Renaud S, Kuntzer T. Favorable outcome of an acute complex regional pain syndrome with immunoglobulin infusions. Clin J Pain 2013;29:e33-e34.

22. Goebel A, Baranowski A, Maurer K, Ghiai A, McCabe C, Ambler G. Intravenous immunoglobulin treatment of the complex regional pain syndrome: a randomized trial. Ann Intern Med 2010;152:152-158.

23. Goebel A, Shenker N, Padfield N, Shoukrey K, McCabe C, Serpell M, Sanders M, Murphy C, Ejibe A, Milligan H, Kelly J, Ambler G. Low-dose intravenous immunoglobulin treatment for complex regional pain syndrome (LIPS): study protocol for a randomized controlled trial. Trials 2014;15:404.

24. Schwartz J, Winters JL, Padmanabhan A, Balogun RA, Delaney M, Linenberger ML, Szczepiorkowski ZM, Williams ME, Wu Y, Shaz BH. Guidelines on the use of therapeutic apheresis in clinical practice-evidence-based approach from the writing committee of the American society for apheresis: the sixth special issue. J Clin Apheresis 2013;28:145-284.

25. Blaes F, Dharmalingam B, Tschernatsch M, Feustel A, Fritz T, Kohr D, Singh P, Kaps M, Szalay G. Improvement of complex regional pain syndrome after plasmapheresis. Eur J Pain (Lond Engl) 2015;19:503-507.

26. Goebel A, Jones S, Oomman S, Callaghan T, Sprotte G. Treatment of long-standing complex regional pain syndrome with therapeutic plasma exchange: a preliminary case series of patients treated in 2008-2014. Pain Med (Malden, Mass) 2014; 15:2163-2164.

27. Hjermstad MJ, Fayers PM, Haugen DF, Caraceni A, Hanks GW, Loge JH, Fainsinger R, Aass N, Kaasa S. Studies comparing numerical rating scales, verbal rating scales, and visual analogue scales for assessment of pain intensity in adults: a systematic literature review. J Pain Symptom Manage 2011;41: 1073-1093.

28. Wallukat G, Munoz Saravia SG, Haberland A, Bartel S, Araujo R, Valda G, Duchen D, Diaz Ramirez I, Borges AC, Schimke I. Distinct patterns of autoantibodies against G-protein-coupled receptors in chagas' cardiomyopathy and megacolon. Their potential impact for early risk assessment in asymptomatic chagas' patients. J Am Coll Cardiol 2010;55:463-468.

29. Strunecka A, Krpejsova L, Palecek J, Macha J, Maturova M, Rosa L, Paleckova A. Transbilayer redistribution of 
phosphatidylserine in erythrocytes of a patient with autoerythrocyte sensitization syndrome (psychogenic purpura). Folia Haematol Int Mag Klin Morphol Blutforsch 1990;117:829-841.

30. Edinger LK, Schwartzman RJ. Gardner-diamond syndrome associated with complex regional pain syndrome. J Dermatol Case Rep 2013;7:10-14.

31. Alexander GM, Peterlin BL, Perreault MJ, Grothusen JR, Schwartzman RJ. Changes in plasma cytokines and their soluble receptors in complex regional pain syndrome. J Pain 2012;13: $10-20$.

32. Lenz M, Uceyler N, Frettloh J, Hoffken O, Krumova EK, Lissek S, Reinersmann A, Sommer C, Stude P, Waaga-Gasser AM, Tegenthoff M, Maier C. Local cytokine changes in complex regional pain syndrome type I (CRPS I) resolve after 6 months. Pain 2013;154:2142-2149.

33. Schlereth T, Drummond PD, Birklein F. Inflammation in CRPS: role of the sympathetic supply. Auton Neurosci 2014;182:102107.

34. Wallukat G, Schimke I. Agonistic autoantibodies directed against G-protein-coupled receptors and their relationship to cardiovascular diseases. Semin Immunopathol 2014;36:351-363.

35. Smith JA, Karalis DG, Rosso AL, Grothusen JR, Hessen SE, Schwartzman RJ. Syncope in complex regional pain syndrome. Clin Cardiol 2011;34:222-225.

36. Li H, Yu X, Liles C, Khan M, Vanderlinde-Wood M, Galloway A, Zillner C, Benbrook A, Reim S, Collier D, Hill MA, Raj SR, Okamoto LE, Cunningham MW, Aston CE, Kem DC. Autoimmune basis for postural tachycardia syndrome. J Am Heart Assoc 2014;3:e000755

37. Vernino S, Low PA, Fealey RD, Stewart JD, Farrugia G, Lennon VA. Autoantibodies to ganglionic acetylcholine receptors in autoimmune autonomic neuropathies. New Engl J Med 2000;343:847-855.

38. Thieben MJ, Sandroni P, Sletten DM, Benrud-Larson LM, Fealey RD, Vernino S, Lennon VA, Shen WK, Low PA. Postural orthostatic tachycardia syndrome: the mayo clinic experience. Mayo Clin Proc 2007;82:308-313.
39. Pasha SF, Lunsford TN, Lennon VA. Autoimmune gastrointestinal dysmotility treated successfully with pyridostigmine. Gastroenterology 2006;131:1592-1596.

40. Vernino S, Hopkins S, Wang Z. Autonomic ganglia, acetylcholine receptor antibodies, and autoimmune ganglionopathy. Auton Neurosci 2009;146:3-7.

41. Dhamija R, Tan KM, Pittock SJ, Foxx-Orenstein A, Benarroch E, Lennon VA. Serologic profiles aiding the diagnosis of autoimmune gastrointestinal dysmotility. Clin Gastroenterol 2008;6: 988-992.

42. Imrich R, Vernino S, Eldadah BA, Holmes C, Goldstein DS. Autoimmune autonomic ganglionopathy: treatment by plasma exchanges and rituximab. Clin Auton Res 2009;19:259-262.

43. Vernino S, Lindstrom J, Hopkins S, Wang Z, Low PA. Characterization of ganglionic acetylcholine receptor autoantibodies. J Neuroimmunol 2008;197:63-69.

44. McKeon A, Lennon VA, Lachance DH, Fealey RD, Pittock SJ. Ganglionic acetylcholine receptor autoantibody: oncological, neurological, and serological accompaniments. Arch Neurol 2009;66:735-741.

45. Hollenbeck R, Black BK, Peltier AC, Biaggioni I, Robertson D, Winton EF, Raj SR. Long-term treatment with rituximab of autoimmune autonomic ganglionopathy in a patient with lymphoma. Arch Neurol 2011;68:372-375.

46. Tambralli A, Beukelman T, Cron RQ, Stoll ML. Safety and efficacy of rituximab in childhood-onset systemic lupus erythematosus and other rheumatic diseases. J Rheumatol 2015;42:541-546.

47. El-Hallak M, Binstadt BA, Leichtner AM, Bennett CM, Neufeld EJ, Fuhlbrigge RC, Zurakowski D, Sundel RP. Clinical effects and safety of rituximab for treatment of refractory pediatric autoimmune diseases. J Pediatr 2007;150:376-382.

48. Iorio R, Damato V, Alboini PE, Evoli A. Efficacy and safety of rituximab for myasthenia gravis: a systematic review and meta-analysis. J Neurol, Oct 42012 (epub ahead of print), PMID: 25308632.

49. Lebrun C, Bourg V, Tieulie N, Thomas P. Successful treatment of refractory generalized myasthenia gravis with rituximab. Eur J Neurol 2009;16:246-250. 